\title{
A cross-sectional study for the mental status and sleep quality among college students in Macao during the COVID-19 pandemic
}

\author{
Xiaoyu Tao ${ }^{1}$, Dong Chen ${ }^{1}$, Yawen Fan ${ }^{1}$, Lanxin Zhang ${ }^{1}$, Houqian Shan ${ }^{1}$, Yi Wei ${ }^{2}$, Xi Yu ${ }^{1}$, Tian Zhong ${ }^{1}$, Ling Wang \\ Corresp., ${ }^{\text {, Sookja Chung }}{ }^{1}$, Yaqin Yu ${ }^{3}$, Ying Xiao ${ }^{\text {Corresp. } 1}$ \\ ${ }^{1}$ Faculty of Medicine, Macau University of Science and Technology, Macao, China \\ 2 School of Public Administration, Jilin University, Changchun, Jilin, China \\ ${ }^{3}$ Faculty of Health Sciences, Zhuhai College of Science and Technology, Zhuhai, Guangdong, China \\ Corresponding Authors: Ling Wang, Ying Xiao \\ Email address: lingwang@must.edu.mo, yxiao@must.edu.mo
}

Objectives: The main focus of this study is to investigate the effect of COVID-19 pandemic on the mental health condition and sleep quality of college students in Macao. In addition, the effects of possible behavioral risk factors, such as alcohol drinking, and the consequence of taking sleeping pills and psychological counselling on mental health and sleep disturbance were determined.

Method: A cross-sectional survey of mental health and sleep quality status, as well as the possible influencing behavioral risk factors was conducted in the college students of Macao in August, 2020 during the COVID-19 pandemic. An online self-report questionnaire survey method was applied to assesss the general demographics and related lifestyle behaviors of students. The general mental health condition and sleep quality were evaluated through the General Health Questionnaire (GHQ-12) and Pittsburgh Sleep Quality Index (PSQI) questionnaires, respectively. The main statistical methods included the Chisquare test, Bonferroni correction, and Pearson correlation. Data analysis was performed using SPSS Version 24.0.

Results: A total of 980 students were investigated in the study, but 977 completed the survey. During the COVID-19 pandemic period, overall college students in Macao were psychologically well adjusted and reported good quality of sleep. However, female students were in poor psychological condition than males $(P<0.05)$, and the students over 20 years of age had poor sleep quality than students aged less than or equal to $20(P<0.05)$. The mental health condition and sleep quality had significant differences among the students in different study majors (both $P<0.05$ ). The general mental health condition and sleep quality of students were associated with certain behaviors, such as drinking alcohol, taking sleeping pills, and searching for help in psychological counselling during the COVID-19 pandemic period.

Conclusions: Poor mental health can be either the consequence or cause of sleep disturbance, which further affects physical health. Therefore, regular assessment of mental health condition and sleep quality of college students is essential during public health emergencies, and appropriate intervention should be provided to the students. 
1 A Cross-sectional Study for the Mental Health Status and Sleep Quality among

2 College Students in Macao during the COVID-19 Pandemic

3

4 Xiaoyu Tao ${ }^{1}$, Dong Chen ${ }^{1}$, Yawen Fan ${ }^{1}$, Lanxin Zhang ${ }^{1}$, Houqian Shan ${ }^{1}$, Yi Wei ${ }^{2}$, Xi Yu ${ }^{1}$, Tian

5 Zhong $^{1}$, Ling Wang $^{1 *}$, Sookja Chung ${ }^{1}$, Yaqin $\mathrm{Yu}^{3}$, Ying Xiao ${ }^{1 *}$

6

8

${ }^{1}$ Faculty of Medicine, Macau University of Science and Technology, Taipa, Macao SAR, China

${ }^{2}$ School of Public Administration, Jilin University, Changchun, Jilin, China

${ }^{3}$ Faculty of Health Sciences, Zhuhai College of Science and Technology, Zhuhai, China

Objectives: The main focus of this study was to investigate the effect of the Coronavirus disease (COVID-19) pandemic on the mental health condition and sleep quality of college students in Macao. In addition, the students' behaviours during the pandemic, such as drinking alcohol, taking sleeping pills, and seeking psychological counselling were analyzed., are suggested of problems with mental health and sleep disturbance.

Method: A cross-sectional survey of mental health and sleep quality status, as well as the possible behavioral risk factors, was conducted on the college students of Macao in August, 2020 during the COVID-19 pandemic. An online self-report questionnaire survey method was applied to assess the general demographics and related lifestyle behaviors of students. The general mental health condition and sleep quality were evaluated through the General Health Questionnaire (GHQ-12) and Pittsburgh Sleep Quality Index (PSQI) questionnaires, respectively. The main statistical methods included the Chi-square test, Bonferroni correction, and Pearson correlation. Data

\footnotetext{
* Corresponding Author:

Prof. Ying Xiao, Faculty of Medicine, Macau University of Science and Technology, Macau SAR, China.

Tel: +85388972607

E-mail: yxiao@must.edu.mo

Dr. Ling Wang, Faculty of Medicine, Macau University of Science and Technology, Macau SAR, China.

Tel: +85388971716

E-mail: lingwang@must.edu.mo
} 
analysis was performed using SPSS Version 24.0.

Results: A total of 980 students were investigated in the study, of which, 977 completed the survey. During the COVID-19 pandemic period, overall college students in Macao were psychologically well adjusted and reported good quality of sleep. However, female students were in poor psychological condition than males $(P<0.05)$. Moreover, the students over 20 years of age had poor sleep quality than students aged less than or equal to $20(P<0.05)$. The significant differences were found among the students in different study majors for the mental health condition and sleep quality (both $P<0.05$ ), which were associated with certain behaviors, such as drinking alcohol, taking sleeping pills, and searching for help in psychological counselling during the COVID-19 pandemic period.

Conclusions: Poor mental health could be either the consequence or cause of sleep disturbance, which might further affect physical health. Therefore, regular assessment of mental health conditions and sleep quality of college students is particularly necessary during public health emergencies, such as the COVID-19 pandemic, and appropriate intervention should be provided to the students.

Keywords: mental health status; sleep quality; college students; COVID-19

\section{INTRODUCTION}

The World Health Organization (WHO) declared an outbreak of COVID-19 on $30^{\text {th }}$ January 2020, which constituted a Public Health Emergency of International Concern (P.H.E.I.C.) (WHO, 2020). The severe acute respiratory syndrome coronavirus 2 (SARS-CoV-2) caused respiratory, cardiovascular and intestinal infections. It is highly contagious and could be transmitted through respiratory droplets and close contacts. The patients' clinical manifestations included asymptomatic symptoms, fever, dry cough, shortness of breath, and even respiratory failure leading to death (Du Toit, 2020; Wiersinga, Rhodes, Cheng, Peacock, \& Prescott, 2020; Zhong et al., 2020). 
The Chinese government implemented prevention and policies to control the spread of the epidemic. In China, it was mandatory for all citizens to wear masks and keep a proper social distance (Horton, 2021). Many regions were closed down, including Macao, which had banned people from entering and leaving the Macao special administrative regions (S.A.R.). For social restriction measures and limited exposure to pathogens, people were advised to workat home and study on-line.

Similar to other major public health emergencies, COVID-19 threatened people's physical health and caused a variety of psychological problems (Lötsch, Schnyder, Goorhuis, \& Grobusch, 2017; Qiu et al., 2020; Talevi et al., 2020). During the COVID-19 pandemic, it was reported that there were more people with higher general mental health scores (GHQ-12) (Ran et al., 2020), which suggested that the mental health conditions of the public became worsen during the COVID19 pandemic period.

At the early stage of COVID-19 pandemics, it did not only affect the physical health, but also, led to emotional tension due to lacking the knowledge of dealing with the disease and urgent physical care, and increased psychological stress and mental illness in the public. Mental health services around the world took urgent measures to address these phenomena (Moreno et al., 2020). Although the community made the effort to provide appropriate psychological guidance to the public, people's mental health was inevitably affected during the pandemic due to the ineffective social communication. College students may be at a higher risk of chronic mental health problems associated with an acute, high-intensity, and uncontrollable stress during the outbreak of the COVID-19, since they are under greater psychological stress resulting from lack of interpersonal communication, active face to face job hunting, online academic studies, and financial difficulties, (Chi et al., 2020). Such pressure may weaken people's sense of happiness and social well being, especially for college students, who showed negative emotions due to the suspension of classes (Zhai \& Du, 2020). It was reported that people aged 18 to 30 and over 60 years old presented with the highest distress index during the COVID-19 peritraumatic condition (Liang et al., 2020b). Disruptions of daily activities, such as group study, leisure, and family communication and social 
life, could also lead to boredom, depression, anxiety, fear, and other negative emotions among college students (Khan et al., 2020; Liang et al., 2020a; Thomas E., 2021). Sleep disorder was an important public health problem. During COVID-19, more people showed insomnia and other sleep disorders (Huang \& Zhu, 2020; Zhou et al., 2020), which may lead to the poor psychological condition of people. Psychological distress among college students has been a concern for public health authorities around the globe. The unsatisfactory sleep also can lead to short and long-term negative impacts on the psychological well-being. It is necessary to investigate the mental health status and sleeping quality, as well as their relationship in college students of Macao during the COVID-19 pandemic and provide some guidelines for preventing mental and sleep disturbance associated with future public health emergencies. The current study analyzed the association between the mental health status, sleep quality, and the behaviors, including alcohol drinking and psychological help-seeking, in college students of Macao during the COVID-19 pandemic period through cross-sectional investigation.

\section{Methodology}

\subsection{Subjects and Procedures}

The online survey was conducted among the college students of Macao during August 2020 before the commencement of the autumn semester. The participants were selected through snowball sampling. The questionnaire was distributed through the Wenjuanxing platform (Changsha Haoxing Information Technology Co., Ltd., Changsha, China). After the informed consent process, the participants completed the questionnaire on the mobile phone by scanning the QR (Quick Response) code via WeChat (a social network APP in China). The questionnaire was filled only once for each registered account holder and was not able to be modified after submission. The Ethical Review Committee of the Zhuhai M.U.S.T. Science Research Academy approval to carry out the study (Ethical Application Ref: MUST-MEC-20200701XY).

\subsection{Measurement}


108

109

110

111

112

113

114

115

116

117

118

119

120

121

122

123

124

125

126

127

128

129

130

\subsubsection{Demographic Variables}

The demographic variables included age ( $\leq 20$ years and $>20$ years), gender (Male and Female), and study major (Humanities \& Arts, Commercial, Law \& Management, Science \& Technology, Medicine \& Health).

\subsubsection{Mental Health}

The 12-item General Health Questionnaire (GHQ-12) is a well-validated, widely used, selfrated surveytoll for detecting psychological morbidity and psychiatric disorders. The Chinese version of the GHQ-12 was used to detect the level of general mental health among the respondents. This questionnaire was developed by Goldberg and revised by scholar Taian Cheng (Cheng \& Williams, 1986; Goldberg David \& Williams, 1988). The common method is binary GHQ scoring (0-0-1-1), which yields a possible score range of $0-12$. Higher scores indicate worse mental health. GHQ-12 scores of 4 and above indicate a tendency towards the psychological disturbance.

\subsubsection{Status of Sleep Quality}

Pittsburgh Sleep Quality Index (PSQI) (Buysse, Reynolds, Monk, Berman, \& Kupfer, 1989; Liu Xianchen, Tang Maoqin, \& Hu Lei, 1996) was used to assess the sleep quality of participants. It consisted of 19 self-evaluation items and 5 other evaluation items. The 19 items constituted 7 demensions, and each component is on a scale from 0 to 3 , with 0 indicating the highest sleep quality and 3 indicating the lowest one (Rasekhi, Pour Ashouri, \& Pirouzan, 1970; Tsai et al., 2005). The cumulative score from the individual dimensions is the total score of PSQI, which ranges from 0 to 21 . The higher the overall score suggests, the worse sleep quality. The scores of 0-4, 5-7, and 8-21 indicate good, general, and poor sleep quality respectively.

\subsubsection{Life behaviors}

The behavioral survey was a self-designed questionnaire based on literature review (Ma \& Lai, 
131 2018; Pretorius et al., 2019; Shi et al, 2020). It allowed to investigate the association between

132

133

134

135

136

137

138

139

140

141

142

143

144

145

146

147

148

149

150

151

152

153

154

155

156

157

mental health and respondents' behaviors, including smoking, alcohol consumption, the use of psychiatric medications, and seeking for psychological services on their own initiative.

\subsection{Data Analysis}

Data were analyzed with SPSS 24.0. Categorical data were analyzed by the chi-square test. Pearson correlation analysis using the demographic variables as independent variables and questionnaire scores as the outcome variable were conducted to identify the factors associated with mental health status. A two-tailed $P<0.05$ was considered statistically significant.

\section{Results}

A total of 977 students completed the questionnaire. The proportion of aged over 20 years was less than the younger group. The ratio of males to females was 1.19. Details are presented in Table 1.

The mental health status was significantly different in various study majors $(P<0.001)$, and students majoring in Humanities \& Arts had poor mental health status than other majors $(P<0.05$, Bonferroni corrected). More details are shown in Table 2. The score of GHQ-12 was significantly higher in female students than that of male ones $(P=0.007)$. On the other hand, 54.86\% of participants had good sleep quality during the COVID-19 pandemic, suggesting that only $45.14 \%$ of participants had poor sleep quality. The sleep quality had significant differences with age $(P=0.004)$ and majors $(P<0.001)$. Participants older than 20 had poor sleep quality. Moreover, students with the major in Medicine \& Health had better sleep quality $(P<0.05$, Bonferroni corrected). The results are shown in Table 3.

During the pandemic, the lifestyle behaviors, such as initiating alcohol drinking, taking sleeping pills, and searching for help in psychological counselling, may affect mental health and sleep quality to a certain extent. The score of GHQ-12 and relieving neurological or psychiatric symptoms by pills and by psychological counselling were related $(P<0.001)$. Furthermore, 
students who used pills or sought counselling had higher GHQ-12 scores and poorer general mental health than those who did not. The students with higher score of PSQI tended to initiate alcohol drinking and take the pills to relieve neurological or psychiatric symptoms $(P<0.05)$, and seek seeking psychological counselling $(P<0.001)$ and poorer sleep quality. The results are summarized in Table 4.

\section{Discussion}

In comparison to the other situation in Mainland China and other countries (Jokela et al., 2020; Lin et al., 2021; Wu et al., 2020), the results of this cross-sectional study showed that the general mental health and sleep quality of college students in Macao were basically good during the COVID-19 pendemic. Only $11.77 \%$ of the participants were rated as having bad general mental health. Such a positive result may be the consequence of the Macao S.A.R. government being actively concerned about the mental health of the populace and the general public during the pandemic. The Macao S.A.R. government created a good anti-pandemic environment by implementing good policies and imposing strict recommendations for the public based on rational scientific data. Generally, the public residents cooperated with the government to fight against the COVID-19 outbreak in Macao and the spread of this disease. At the same time, colleges in Macao attached importance to the psychological changes of students, ensured that college students could get psychological care and counselling in time when needed during the pandemic (New Coronavirus Infection Strain Coordination Center, 2021).

General mental health status was strongly correlated with gender during the COVID-19 pendemic in the investigated students $(P=0.007)$. Compared with males, females had poorer general mental health, which was consistent with previous research results (Wu et al., 2020). It could be seen that, females had more psychological stress and were more distressed during the pandemic; there was a significant difference between general mental health status and study major. The students who majored in Humanities \& Art were under high psychological pressure. The first reason was that they might have less knowledge of the COVID-19 pandemic due to their 
professional background (Peng et al., 2020). The second possible reason was that the pandemic had increased people's social distance, and their profession required more interpersonal communication. Medical students had solid knowledge of the COVID-19 pandemic prevention, so their psychological pressure was lighter (Zeng, Chen, Wang, Zhang, \& Deng, 2019).

The lack of space for physical activity, limited social interaction and psychological stress associated with fear of infection after the COVID-19 outbreak may reduce the sleep quality in college students and increase their negative emotion. The COVID-19 outbreak had an indirect impact on the mental health of young people, which could be mediated by sleep quality (Zhang, Zhang, Ma, \& Di, 2020). Unlike previous study (S. J. Zhou et al., 2020), there was no statistically significant gender difference in sleep quality in the college students of Macao. However, college students over the age of 20 (almost junior and senior) showed a poorer sleep quality, possibly due to that the students in senior year face more pressure from making plans for higher degree program studies or employment issues (Chi et al., 2020). In addition, online courses may affect the quality of communication and interaction among students and their teachers. In some cases, the completion of the final year or higher degree thesis could not be completed as expected due to face to face execution of the project and discussion of research data. During the COVID19 outbreak, many businesses closed down leading to higher unemployment. Graduates faced more competition for fewer positions when looking for jobs. Interestingly, Medical students' sleep quality was better than other major students, possibly because medical students are better informed about how to protect themselves during the pandemic, less worried and stressed (Zeng et al., 2019).

Students, who had poorer sleep quality and mental health issues initiated drinking alcohol, taking pills, and seeking internet counselling to relieve neurological or psychiatric symptoms. The unsupervised, self-initiated intervention against mental and sleep disorders of students can lead to more desastrous outcomes. To maintain the good mental health of college students, it was recommended that the colleges' health care unit should actively investigate the mental health of students during the pandemic, especially for senior students, and encourage students to seek psychological counselling during the pandemic. According to a survey, students who had 
212 undergone psychological counselling during the epidemic were generally in a poor state of mental

213 health (Liang et al., 2020). Students could also appropriately use the internet for consultation.

214 However, young people had different evaluations of online psychological counselling services

215 (Pretorius et al., 2019). It is recommended to enrich the resources of internet psychology and 216 improve their qualities. In addition, mental health clinics of schools should identify students 217 seeking alcohol drinking or seeking pills to relieve their mental state and investigate the students' 218 mental problems as early as possible to provide face to face or online counselling. Particularly 219 during the COVID-19 pandemic, students should be recommended to maintain healthy lifestyle with good living habits, which may improve mental state and sleep quality.

\section{Conclusions}

Poor mental health could be either the consequence or cause of sleep disturbance, which might further affect physical health. Therefore, regular assessment of mental health conditions and sleep quality of college students is particularly necessary during public health emergencies, such as the COVID-19 pandemic, and appropriate intervention should be provided to the students.

\section{Limitations}

The current study had several limitations. First, it was a cross-sectional survey, which could not explain the cause-and-effect relationship between the COVID-19 pandemic and mental health status or sleep quality. Then, the study population was not a random sample and might not avoid bias completely in subject selection. Lastly, the questionnaire was finished in the form of a selfreport; therefore, some of the answers might be subjective.

Funding: This study was supported by the Higher Education Fund of the Macao SAR Government analysis, decision to publish, or preparation of the manuscript. 
239 Acknowledgements: We would like to thank all the participants who participated in this study. 240

241 Conflicts of Interest: The authors declare no conflict of interest.

243 Ethical Approval Information: This study was supported by the Higher Education Fund of the 244 Macao SAR Government (Grant No. HSS-MUST-2020-6). The funders had no role in study 245 design, data collection and analysis, decision to publish, or preparation of the manuscript.

Participants' Consent for Publication: Consent form was obtained from every participant in our 248 study. Participants, who agreed to participate in the survey, were instructed to click the "Agree to 249 participate" button and then directed to complete the anonymous self-report questionnaire. 
250

251

252

253

254

255

256

257

258

259

260

261

262

263

264

265

266

267

268

269

270

271

272

273

274

275

276

277

278

279

280

281

282

283

284

285

286

287

288

289

290

\section{REFERENCES}

1. Almeida, M., Shrestha, A. D., Stojanac, D., \& Miller, L. J. (2020). The impact of the COVID-19 pandemic on women's mental health. Archives of women's mental health, 23(6), 741-748. doi: 10.1007/s00737-020-010922..

2. Buysse, D. J., Reynolds, C. F., 3rd, Monk, T. H., Berman, S. R., \& Kupfer, D. J. (1989). The Pittsburgh Sleep Quality Index: a new instrument for psychiatric practice and research. Psychiatry Res, 28(2), 193-213. doi:10.1016/0165-1781(89)90047-4

3. Cheng \& Williams (1986) Cheng TA, Williams P. The design and development of a screening questionnaire (CHQ) for use in community studies of mental disorders in Taiwan. Psychological Medicine. 1986;16:415-422. doi: $10.1017 / \mathrm{s} 0033291700009247$.

4. Chi, X., Becker, B., Yu, Q., Willeit, P., Jiao, C., Huang, L., Hossain, M. M., Grabovac, I., Yeung, A., Lin, J., Veronese, N., Wang, J., Zhou, X., Doig, S. R., Liu, X., Carvalho, A.F., Yang, L., Xiao, T., Zou, L., Fusar-Poli, P., \&Solmi, M. (2020). Prevalence and Psychosocial Correlates of Mental Health Outcomes Among Chinese College Students During the Coronavirus Disease (COVID-19) Pandemic. Front Psychiatry, 11, 803. doi:10.3389/fpsyt.2020.00803

5. Du Toit, A. (2020). Outbreak of a novel coronavirus. Nature Reviews Microbiology, 18(3), 123-123. doi:10.1038/s41579-020-0332-0

6. Goldberg David \& Williams (1988) Goldberg David P, Williams P. A user's guide to the general health questionnaire. NFER-Nelson; Windsor: 1988.

7. Horton, R. (2021). Offline: 2019-nCoV—“A desperate plea” - The Lancet. doi:doi:10.1016/S01406736(20)30299-3

8. Hu, N., Ma, Y., He, J., Zhu, L., \& Cao, S. (2020). Alcohol consumption and incidence of sleep disorder: A systematic review and meta-analysis of cohort studies. Drug and Alcohol Dependence, 217, 108259. doi:https://doi.org/10.1016/j.drugalcdep.2020.108259

9. Huang, Y., \& Zhu, M. (2020). Increased Global PSQI Score Is Associated with Depressive Symptoms in an Adult Population from the United States. Nature and science of sleep, 12, 487-495. doi:10.2147/NSS.S256625

10. Jokela, M., García-Velázquez, R., Komulainen, K., Savelieva, K., Airaksinen, J., \& Gluschkoff, K. (2020). Specific symptoms of the General Health Questionnaire (GHQ) in predicting persistence of psychological distress: Data from two prospective cohort studies. Journal of Psychiatric Research.

11. Khan, A. H., Sultana, M. S., Hossain, S., Hasan, M. T., Ahmed, H. U., \& Sikder, M. T. (2020). The impact of COVID-19 pandemic on mental health \& wellbeing among home-quarantined Bangladeshi students: A crosssectional pilot study. Journal of affective disorders, 277, 121-128. doi:10.1016/j.jad.2020.07.135

12. Liang, S.W., Chen, R.N., Liu, L.L., Li, X.G., Chen, J.B., Tang, S.Y., \& Zhao, J.B. (2020). The psychological impact of the COVID-19 epidemic on Guangdong college students: the difference between seeking and not seeking psychological help. Frontiers in Psychology, 11(2231). doi:10.3389/fpsyg.2020.02231

13. Lin, Y., Liu, S., Li, S., Zuo, H., \& Zhang, B. (2021). Relationships between the changes in sleep patterns and sleep quality among Chinese people during the 2019 coronavirus disease outbreak. Sleep Med. doi:10.1016/j.sleep.2021.01.021

14. Liu X.C., Tang, M. Q., \& Hu L., (1996). Reliability and validity of the Pittsburgh sleep quality index. Chinese Journal of Psychiatry(02), 103-107. (in Chinese)

15. Lötsch, F., Schnyder, J., Goorhuis, A., \& Grobusch, M. P. (2017). Neuropsychological long-term sequelae of 
Ebola virus disease survivors - A systematic review. Travel Med Infect Dis, 18, 18-23. doi:10.1016/j.tmaid.2017.05.001

16. Ma, C. M., \& Lai, C. C. (2018). Mental health profile and health-related behavior among Hong Kong Chinese university students. Health Psychology Open, 5(2), 2055102918786869. doi:10.1177/2055102918786869

17. Moreno, C., Wykes, T., Galderisi, S., Nordentoft, M., Crossley, N., Jones, N., Cannon, M., Correll, C. U., Byrne, L., Carr, S., Chen, E. Y. H., Gorwood, P., Johnson, S., Kärkkäinen, H., Krystal, J. H., Lee, J., Lieberman, J., López-Jaramillo, C., Männikkö, M., Phillips, M. R., Uchida, H., Vieta, E., Vita, A., \& Arango, C. (2020). How mental health care should change as a consequence of the COVID-19 pandemic. Lancet Psychiatry, 7(9), 813824. doi:10.1016/s2215-0366(20)30307-2

18. New Coronavirus Infection Strain Coordination Center. (2021). Suspended updating and implementation of the Management Recommendations for the Prevention of New Coronary Virus Pneumonia - Collective Festivals, Recreation and Wellness Activities. Retrieved from https://www.gov.mo/zh-hant/news/351965/ (in Chinese)

19. World Health Organization (2020). Archived: WHO Timeline - COVID-19. Retrieved from https://www.who. int/news/item /27-04-2020-who-timeline---covid-19

20. Peng, Y., Pei, C., Zheng, Y., Wang, J., Zhang, K., Zheng, Z., \& Zhu, P. (2020). A cross-sectional survey of knowledge, attitude and practice associated with COVID-19 among undergraduate students in China. BMC public health, 20(1), 1-8.

21. Pretorius, C., Chambers, D., Cowan, B., \& Coyle, D. (2019). Young people seeking help online for mental health: cross-sectional survey study. JMIR Ment Health, 6(8), e13524. doi:10.2196/13524

22. Qiu, J., Shen, B., Zhao, M., Wang, Z., Xie, B., \& Xu, Y. (2020). A nationwide survey of psychological distress among Chinese people in the COVID-19 epidemic: implications and policy recommendations. General psychiatry, 33(2), e100213. doi:10.1136/ gpsych-2020-100213. (Accession No. 32215365)

23. Ran, M. S., Gao, R., Lin, J. X., Zhang, T. M., Chan, S. K. W., Deng, X. P., Zhang, B. Z., Zhang, X. F., Huang, G. P., Pu, D. S., Bai, J. Z., Xu, L. X., \& Liu, B. (2020). The impacts of COVID-19 outbreak on mental health in general population in different areas in China. Psychol Med, 1-10. doi:10.1017/s0033291720004717

24. Rasekhi, S., Pour Ashouri, F., \& Pirouzan, A. (1970). Effects of sleep quality on the academic performance of undergraduate medical students. Health Scope, 5(3), e31641. doi:10.17795/jhealthscope-31641

25. Shi, W., Shen, Z., Wang, S., \& Hall, B. J. (2020). Barriers to professional mental health help-seeking among chinese adults: a systematic review. Frontiers in Psychiatry, 11(442). doi:10.3389/fpsyt.2020.00442

26. Talevi, D., Socci, V., Carai, M., Carnaghi, G., Faleri, S., Trebbi, E., di Bernardo, A., Capelli, F., \&Pacitti, F. (2020). Mental health outcomes of the CoViD-19 pandemic. Riv Psichiatr, 55(3), 137-144. doi: $10.1708 / 3382.33569$

27. Tran, T. D., Tran, T., \& Fisher, J. (2012). Validation of three psychometric instruments for screening for perinatal common mental disorders in men in the north of Vietnam. Journal of Affective Disorders, 136(1), 104109. doi:https://doi.org/10.1016/j.jad.2011. 08.012

28. Tsai, P. S., Wang, S. Y., Wang, M. Y., Su, C. T., Yang, T. T., Huang, C. J., \& Fang, S. C. (2005). Psychometric evaluation of the Chinese version of the Pittsburgh Sleep Quality Index (CPSQI) in primary insomnia and control subjects. Qual Life Res, 14(8), 1943-1952. doi:10.1007/s11136-005-4346-x

29. Wiersinga, W. J., Rhodes, A., Cheng, A. C., Peacock, S. J., \& Prescott, H. C. (2020). Pathophysiology, Transmission, Diagnosis, and Treatment of Coronavirus Disease 2019 (COVID-19): A Review. Jama, 324(8), 782-793. doi:10.1001/jama.2020.12839 
332

333

334

335

336

337

338

339

340

341

342

343

344

345

346

347

348

349

350

30. Wu, D., Yu, L., Yang, T., Cottrell, R., Peng, S., Guo, W., \& Jiang, S. (2020). The impacts of uncertainty stress on mental disorders of Chinese college students: Evidence from a nationwide study. Frontiers in Psychology, 11, 243. doi: 10.3389/fpsyg.2020.00243..

31. Thomas E.,. (2021). Coronavirus report: impact on young people with mental health needs. Youngminds.org.uk. https:/youngminds.org.uk/about-us/reports/coronavirus-impact-on-young-people-with-mental-health-needs/

32. Zeng, W., Chen, R., Wang, X., Zhang, Q., \& Deng, W. (2019). Prevalence of mental health problems among medical students in China: A meta-analysis. Medicine, 98(18), e15337. doi:10.1097/md.0000000000015337

33. Zhai, Y., \& Du, X. (2020). Addressing collegiate mental health amid COVID-19 pandemic. Psychiatry Res, 288, 113003. doi:10.1016/j.psychres.2020.113003

34. Zhang, Y., Zhang, H., Ma, X., \& Di, Q. (2020). Mental health problems during the COVID-19 pandemics and the mitigation effects of exercise: a longitudinal study of college students in China. Int J Environ Res Public Health, 17(10). doi:10.3390/ijerph17103722

35. Zhong, B.L., Luo, W., Li, H.M., Zhang, Q.Q., Liu, X.G., Li, W.T., \& Li, Y. (2020). Knowledge, attitudes, and practices towards COVID-19 among Chinese residents during the rapid rise period of the COVID-19 outbreak: a quick online cross-sectional survey. International Journal of Biological Sciences, 16(10), 1745-1752. doi:10.7150/ijbs.45221

36. Zhou, S.J., Wang, L.L., Yang, R., Yang, X.J., Zhang, L.G., Guo, Z.C., Chen, J. C., Wang, J. Q., \& Chen, J.X. (2020). Sleep problems among Chinese adolescents and young adults during the coronavirus-2019 pandemic. Sleep medicine, 74, 39-47. doi: 10.1016/j.sleep.2020.06.001 
Table $\mathbf{1}$ (on next page)

Demographic_characteristics_of the_participant 
Table 1. Demographic characteristics of the participant $(n=977)$

\begin{tabular}{lc}
\hline \multicolumn{1}{c}{ Items } & $\mathrm{n}(\%)$ \\
\hline Age (year) & $594(60.80)$ \\
$\leq 20$ & $383(39.20)$ \\
$>20$ & \\
Gender & $530(54.25)$ \\
Male & $447(45.75)$ \\
Female & \\
Major & $238(24.36)$ \\
Humanities and arts & $286(29.27)$ \\
Commercial, law and management & $236(24.16)$ \\
Science and technology & $217(22.21)$ \\
\hline
\end{tabular}

2 
Table 2 (on next page)

GHQ-12_scores_of_the_participant 
Table 2. GHQ-12 scores of the participant $(n=977)$

\begin{tabular}{lllll}
\hline & Good $(\%)$ & Poor $(\%)$ & $\chi^{2}$ & $P$ \\
\hline Age & & & \\
$\leq 20$ & $310(87.32)$ & $45(12.68)$ & 0.393 & 0.531 \\
$>20$ & $552(88.75)$ & $70(11.25)$ & & \\
Gender & & & & \\
Male & $408(91.28)$ & $39(8.72)$ & & \\
Female & $454(85.66)$ & $76(14.34)$ & & \\
Major & & & & \\
Humanities \& Arts & $195(81.93)$ & $43(18.07)$ & & \\
Commercial, Law \& Management & $246(86.01)$ & $40(13.99)$ & & \\
Science \& Technology & $221(93.64)$ & $15(6.36)$ & & \\
Medicine \& Health & $200(92.17)$ & $17(7.83)$ & & \\
\hline
\end{tabular}


Table 3 (on next page)

PSQI_scores_of_the_participant 
1

\begin{tabular}{lccccc}
\multicolumn{6}{c}{ Table 3. PSQI scores of the participant (n=977) } \\
\hline Good (\%) & General (\%) & poor (\%) & $\chi^{2}$ & $P$ \\
\hline Age & & & & & \\
$\leq 20$ & $350(58.92)$ & $162(27.27)$ & $82(13.80)$ & 11.072 & 0.004 \\
$>20$ & $186(48.56)$ & $122(1.85)$ & $75(19.58)$ & & \\
Gender & & & & & \\
Male & $263(58.84)$ & $120(26.85)$ & $64(14.32)$ & 5.348 & 0.069 \\
Female & $273(51.51)$ & $164(30.94)$ & $93(17.55)$ & & \\
Major & & & & & \\
Humanities \& Arts & $109(45.80)$ & $82(34.45)$ & $47(19.75)$ & 21.602 & 0.001 \\
Commercial, Law \& Management & $155(54.20)$ & $74(25.87)$ & $57(19.93)$ & & \\
Science \& Technology & $134(56.78)$ & $73(30.93)$ & $29(12.29)$ & & \\
Medicine \&Health & $138(63.59)$ & $55(25.35)$ & $24(11.06)$ & & \\
\hline
\end{tabular}




\section{Table 4 (on next page)}

Correlation_between_life_behavior_and_general_mental_health_\&_sleep_quality_during_ the_pandemic_in_the_subjects 
1 Table 4. Correlation between life behavior and general mental health \& sleep quality during the pandemic in the subjects

\begin{tabular}{|c|c|c|c|c|c|c|c|c|c|}
\hline & & \multirow[t]{2}{*}{ GHQ-12 } & \multirow[t]{2}{*}{ PSQI } & \multicolumn{6}{|c|}{ The behaviors during the COVID-19 pandemic } \\
\hline & & & & $\begin{array}{l}\text { The original cigarette } \\
\text { consumption has increased }\end{array}$ & $\begin{array}{l}\text { Try to start } \\
\text { smoking }\end{array}$ & $\begin{array}{l}\text { The original alcohol } \\
\text { consumption has increased }\end{array}$ & $\begin{array}{l}\text { Try to start } \\
\text { drinking }\end{array}$ & $\begin{array}{l}\text { Relieve neurological or } \\
\text { psychiatric symptoms by pills }\end{array}$ & $\begin{array}{l}\text { Seek psychologica } \\
\text { counselling }\end{array}$ \\
\hline \multirow[t]{2}{*}{ GHQ-12 } & Pearson Correlation Coefficient & 1 & 0.259 & -0.274 & -0.032 & -0.008 & -0.034 & -0.169 & -0.318 \\
\hline & $P$ & & 0.073 & 0.057 & 0.327 & 0.936 & 0.315 & $<0.001$ & $<0.001$ \\
\hline \multirow[t]{2}{*}{ PSQI } & Pearson Correlation Coefficient & 0.259 & 1 & 0.011 & -0.023 & -0.020 & -1.00 & -0.262 & -0.259 \\
\hline & $P$ & 0.073 & & 0.940 & 0.476 & 0.844 & 0.003 & $<0.001$ & $<0.001$ \\
\hline
\end{tabular}

2 
